\title{
Coronal Properties of Swift/BAT-selected Seyfert 1 AGNs Observed with NuSTAR
}

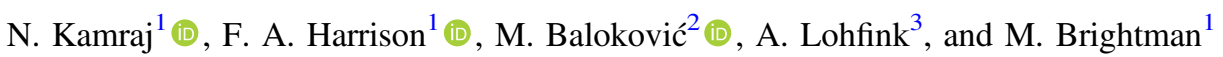 \\ ${ }^{1}$ Cahill Center for Astronomy and Astrophysics, California Institute of Technology, Pasadena, CA 91125, USA; nkamraj@caltech.edu \\ ${ }^{2}$ Harvard-Smithsonian Center for Astrophysics, 60 Garden Street, Cambridge, MA 02138, USA \\ ${ }^{3}$ Department of Physics, Montana State University, 211 Montana Hall, Bozeman, MT 59717, USA \\ Received 2018 April 20; revised 2018 August 23; accepted 2018 August 24; published 2018 October 19
}

\begin{abstract}
The NUSTAR observatory, with its high sensitivity in hard X-rays, has enabled detailed broadband modeling of the X-ray spectra of active galactic nuclei (AGNs), thereby allowing constraints to be placed on the high-energy cutoff of the X-ray coronal continuum. We investigate the spectral properties of a sample of $46 \mathrm{NuSTAR}$-observed Seyfert 1 AGNs selected from the Swift/Burst Alert Telescope 70 month hard X-ray survey. Our measurements of the highenergy cutoff of the continuum from modeling the NuSTAR X-ray spectra are used to map out the temperaturecompactness $(\theta-l)$ plane for AGN coronae. We find that most of the coronae lie clustered near the boundary for runaway pair production, suggesting that annihilation and pair production act to regulate the temperature of the corona. We discuss the implications of coronae whose high-energy cutoff may indicate a low coronal temperature on the heating and thermalization mechanisms in the corona.
\end{abstract}

Key words: black hole physics - galaxies: active - X-rays: galaxies

\section{Introduction}

The continuum X-ray emission from active galactic nuclei (AGNs) is believed to originate in a hot, compact corona located above the accretion disk (e.g., Haardt \& Maraschi 1993). Compton upscattering of UV and optical photons from the inner accretion disk by coronal electrons produce a power-law-like X-ray continuum, with a cutoff at energies determined by the electron temperature $T_{e}$ (e.g., Rybicki \& Lightman 1979; Zdziarski et al. 2000). The shape of the coronal continuum is sensitive to properties such as the seed photon field, electron temperature, optical depth, and observer viewing angle. The observed rapid variability of the $2-10 \mathrm{keV}$ emission in many AGNs, combined with X-ray spectral timing and reverberation mapping, strongly indicates that the corona is physically compact, of the order 3-10 gravitational radii (Fabian et al. 2009, 2015; Kara et al. 2013; Emmanoulopoulos et al. 2014). The gravitational radius is defined to be $G M_{\mathrm{BH}} / c^{2}$, where $M_{\mathrm{BH}}$ is the supermassive black hole mass. Such radiatively compact sources can exchange significant energy between particles and photons, with the compactness characterized by the dimensionless parameter $l$ (Guilbert et al. 1983), defined as

$$
l=4 \pi \frac{m_{p}}{m_{e}} \frac{R_{g}}{R} \frac{L}{L_{E}}
$$

where $m_{p}$ and $m_{e}$ are the proton and electron mass, respectively, $R_{g}$ is the gravitational radius, $R$ is the source radius, $L$ is the source luminosity, and $L_{\mathrm{E}}$ is the Eddington luminosity. The electron temperature $T_{e}$ can also be characterized by the dimensionless parameter $\theta=k_{\mathrm{B}} T_{e} / m_{e} c^{2}$, where $k_{\mathrm{B}}$ is the Boltzmann constant. For sufficiently energetic photons, photon-photon collisions can lead to electron-positron pair production in the corona (Svensson 1982; Guilbert et al. 1983; Zdziarski 1985). At high coronal temperatures, when the Wien tail of the power-law spectrum extends above $2 m_{e} c^{2}$, pair production can quickly become a runaway process, exceeding annihilation (Svensson 1984). This will limit any further rise in temperature, thus acting as an l-dependent thermostat (Svensson 1984; Zdziarski 1985; Stern et al. 1995).
The NuSTAR observatory (Harrison et al. 2013), being the first focusing hard X-ray telescope in orbit, has enabled detailed, high signal-to-noise spectra to be obtained in the $3-79 \mathrm{keV}$ band for many local AGNs. NUSTAR spectral modeling can thus place constraints on the spectral photon index and high-energy cutoff of the coronal X-ray continuum, enabling robust estimates of $l$ and $\theta$. One of the primary goals of the NUSTAR mission is to perform an extragalactic survey of the hard X-ray sky in order to characterize the AGN population. We define hard X-rays as photons with energies $>10 \mathrm{keV}$. As part of its Extragalactic Legacy Surveys program, ${ }^{4}$ the NUSTAR observatory has performed snapshot $\sim 20 \mathrm{ks}$ observations of local AGNs detected in the all-sky survey with the Burst Alert Telescope (BAT) instrument on board the Neil Gehrels Swift Observatory (Gehrels et al. 2004; Baumgartner et al. 2013). Though previous work has provided broad constraints on the high-energy cutoff for samples of bright AGNs, tight constraints for particular AGNs only became available recently thanks to NuSTAR (e.g., Ballantyne et al. 2014; Brenneman et al. 2014; Marinucci et al. 2014; Baloković et al. 2015; Fabian et al. 2015). The 100-fold increase in sensitivity of the NuSTAR telescope compared to the Swift/BAT instrument enables robust spectral modeling with a minimal NUSTAR exposure of $\sim 20 \mathrm{ks}$. With even longer exposure NuSTAR observations, it is possible to obtain tight limits on X-ray spectral parameters and perform reverberation mapping measurements of coronal size.

In this paper, we study a sample of $46 \mathrm{Swift} / \mathrm{BAT}$-selected Seyfert 1 (Sy1) AGNs observed with NuSTAR, in order to map out the location of these sources on the temperature-compactness $(\theta-l)$ diagram for AGN coronae. We do not include Swift/XRT data in our spectral modeling as the limited data quality of available simultaneous Swift/XRT data introduces difficulties in obtaining constraints on parameters such as the cutoff energy. The complexity of features in soft X-ray spectra would ideally require high signal-to-noise ratio, simultaneous spectra from soft X-ray telescopes with larger collecting area to model robustly, which are currently unavailable for the targets in our sample. In Section 2, we discuss the sample used in this study, the data reduction, and

\footnotetext{
4 https://www.nustar.caltech.edu/page/legacy_surveys
} 


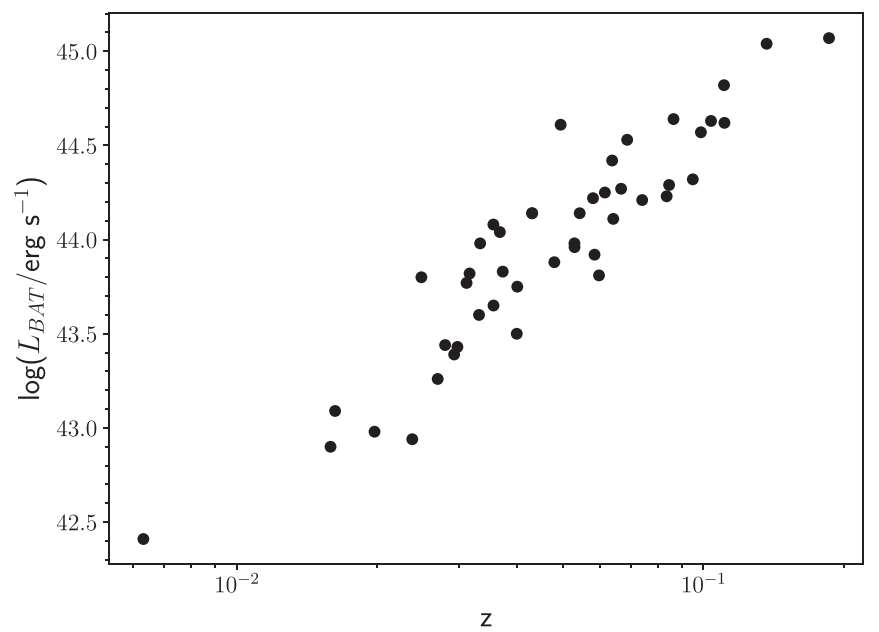

Figure 1. Redshift-luminosity distribution for NuSTAR-observed Sy1 AGNs selected from the Swift/BAT 70 month hard X-ray catalog.

analysis procedures adopted. Observational details of our AGN sample are presented in the Appendix. In Section 3, we present our results and discuss the heating and cooling mechanisms operating in the corona. We discuss future, deeper NuSTAR observations of AGNs in our sample with potential cutoffs in the NuSTAR band in Section 4, and present a summary in Section 5. In this work, all uncertainties were calculated at the $90 \%$ confidence level and standard values of the cosmological parameters $\left(h_{0}=0.7\right.$, $\Omega_{\Lambda}=0.7, \Omega_{m}=0.3$ ) were used to calculate distances.

\section{Sample, Data Reduction, and Analysis}

\subsection{Sample of Seyfert 1 AGNs}

We selected our sample from AGNs identified in the Swift/ BAT 70 month hard X-ray catalog (Gehrels et al. 2004; Baumgartner et al. 2013). From the full catalog, we selected NuSTARobserved AGNs with known redshifts and classified as Sy1 from optical hydrogen emission line measurements, or from available data from the NASA/IPAC Extragalactic Database (NED). The full list of AGNs included in our study, along with their NuSTAR observation details, may be found in the Appendix. Figure 1 shows the location of our sources on the redshift-luminosity plane, with the luminosity values determined from the Swift/BAT fluxes in the 14-195 keV range. We confirmed sources at high redshift to not be beamed AGN or blazar candidates from observations of their optical spectra and cross-matching with the Roma Blazar Catalog (Massaro et al. 2009). We found two sources that were misclassified from NED and were removed from our sample. We excluded nine sources from our original sample due to lack of constraints on the high-energy cutoff from spectral fitting. Our final sample consists of 46 Sy1 AGNs at $0.003<z<0.2$.

In Figure 2, we present the distributions of Swift/BAT fluxes, luminosities, and redshifts for both our sample and the Sy1 classified sources from the Swift/BAT 70 month catalog. We find that our sample is statistically representative of the Sy1 population from the Swift/BAT 70 month catalog, with the mean and median values overlapping between our sample and the parent Swift/BAT sample. We further applied a two-sample Kolmogorov-Smirnov test and found the $\mathrm{K}-\mathrm{S}$ test statistic to be 0.1 or lower, and the $p$-value above $60 \%$ for all three distributions, thus confirming that the distributions are consistent between our sample and the larger Swift/BAT sample of Syls.
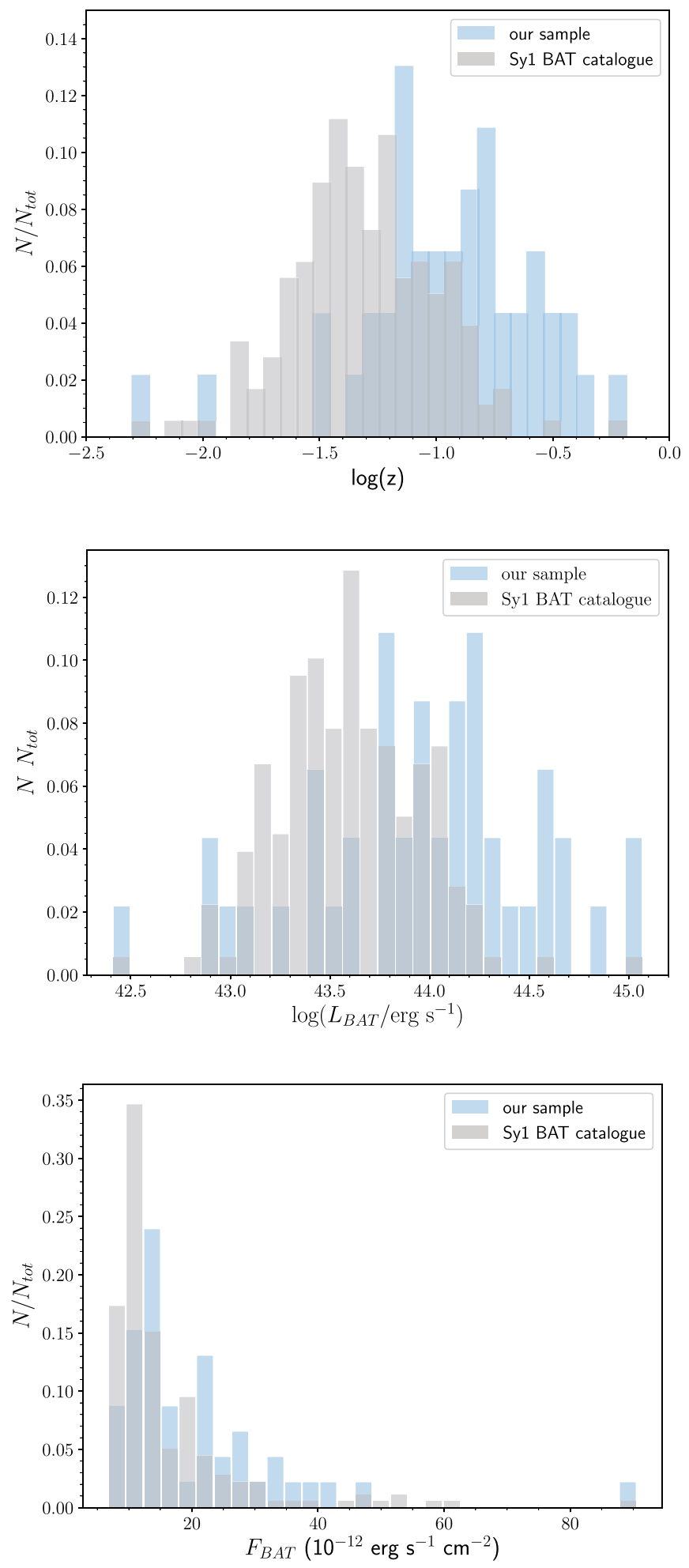

Figure 2. Distributions of redshifts, Swift/BAT 70 month X-ray catalog luminosities, and fluxes for both our sample and the Sy1 classified sources from the Swift/BAT catalog. For clarity, sources with $L_{\mathrm{BAT}}<10^{40} \mathrm{erg} \mathrm{s}^{-1}$ and $F_{\mathrm{BAT}}>1 \times 10^{-10} \mathrm{erg} \mathrm{s}^{-1} \mathrm{~cm}^{-2}$ were omitted from the plots.

\subsection{NuSTAR Observations and Data Reduction}

Roughly once per week since its start of science operations in 2013, the NUSTAR satellite has been obtaining $\sim 20 \mathrm{ks}$ observations in the 3-79 keV band of AGNs selected from the Swift/BAT 70 month hard X-ray catalog (Baumgartner 
et al. 2013). We performed reduction of raw event data from both NUSTAR modules, FPMA, and FPMB (Harrison et al. 2013), using the NuSTAR Data Analysis Software (NuSTARDAS; version 1.2.1), distributed by the NASA High Energy Astrophysics Archive Research Center (HEASARC) within the HEASOFT package (version 6.16). We took instrumental responses from the NuSTAR calibration database (CALDB; version 20160502). Raw event data were cleaned and filtered for South Atlantic Anomaly passages using the nupipeline module. We extracted source and background energy spectra from the calibrated and cleaned event files using the nuproducts module. Detailed information on these data reduction procedures can be found in the NUSTAR Data Analysis Software Guide (Perri et al. 2017). An extraction radius of $30^{\prime \prime}$ was used for both the source and background regions. We extracted the background spectrum from sourcefree regions of the image, and away from the outer edges of the field of view, which have systematically higher background. The spectral files were rebinned using the HEASOFT task grppha to give a minimum of 20 photon counts per bin. For multiple observations of the same source, we coadded spectra using the HEASOFT task addspec.

\subsection{Spectral Modeling}

We performed spectral modeling of the NuSTAR data in the 3-79 keV band for each source in our sample using XSPEC v12.8.2 (Arnaud 1996). We used $\chi^{2}$ statistics for all model fitting and error estimation. We adopted cross sections from Verner et al. (1996) and solar abundances from Wilms et al. (2000). In all our modeling we include a cross-correlation constant between FPMA and FPMB to account for slight differences in calibration (Madsen et al. 2015).

We fit each spectrum with an absorbed power-law model with a high-energy cutoff, $E_{\text {cut }}$. The slope of the power-law continuum is characterized by the photon index, $\Gamma$. It is assumed that the intrinsic continuum intensity is proportional to $E^{-\Gamma} \exp \left(-E / E_{\text {cut }}\right)$. In XSPEC notation, the model used is TBabs $\times$ zwabs $\times$ cutoffpl, where the component TBabs models Galactic absorption, which is fixed to a typical Galactic column density of $7.6 \times 10^{21} \mathrm{~cm}^{-2}$ (Kalberla et al. 2005). We found that freezing the Galactic column density did not have any significant effect on the fit results, as spectral modeling over the hard X-ray band is relatively insensitive to this parameter. The redshifted component zwabs accounts for absorption by the host galaxy.

Where an $\mathrm{Fe} \mathrm{K} \alpha$ emission line feature was observed in the spectra at $6.4 \mathrm{keV}$, we added an additive zgauss Gaussian line component to the absorbed power-law model. We note that two objects out of our sample required fitting with an $\mathrm{Fe} \mathrm{K} \alpha$ line: Mrk 595 and RBS 1037. In addition, we test for the presence of spectral features due to reprocessing by adding a pexrav component (Magdziarz \& Zdziarski 1995). We fixed elemental abundances to solar and kept the inclination angle fixed at the default value of $60^{\circ}$. We found that the reduced $\chi^{2}$ values and best-fit parameters from modeling with pexrav were similar to those from fitting an absorbed cutoff power-law for the majority of the sources in our sample, indicating that the addition of a reflection component does not significantly modify fit results and thus is not required by the data. Furthermore, we found that the null hypothesis probability exceeds $50 \%$ for many of our sources when fitting with an absorbed cutoff power-law; we found the mean null hypothesis probability of our sample to be $43 \%$. We note that we chose a reflection model for one source (2MASX

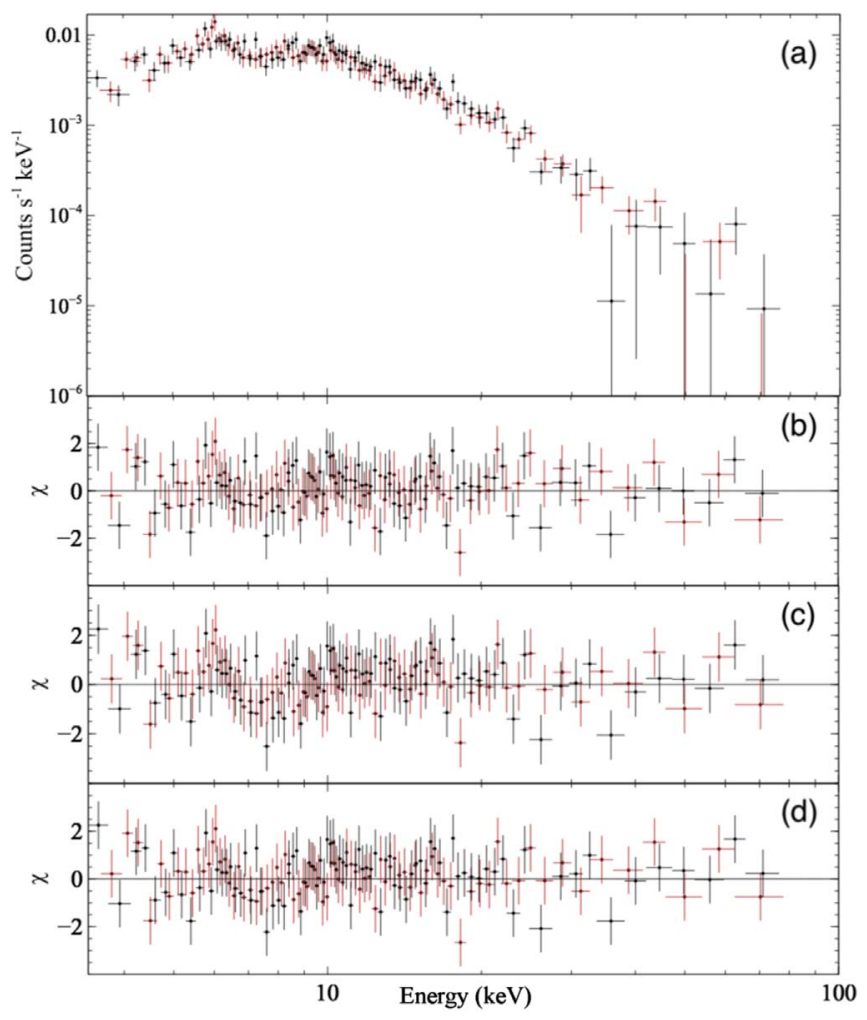

Figure 3. (a) NUSTAR hard X-ray spectrum of a candidate low-cutoff AGN 2MASX J19301380+3410495, alongside fit residuals for (b) an absorbed power-law model $\left(\chi^{2} /\right.$ dof $\left.=155.1 / 161\right)$, (c) an absorbed power-law model with a high-energy cutoff $\left(\chi^{2} /\right.$ dof $\left.=144.4 / 160\right)$, and $(d)$ an absorbed cutoff power-law with reflection modeled via pexrav $\left(\chi^{2} /\right.$ dof $\left.=138 / 160\right)$. Black points correspond to FPMA data, while points in red correspond to FPMB.

$\mathrm{J} 19301380+3410495)$ due to best-fit parameters such as the photon index being more physically reasonable compared to the absorbed cutoff power-law model. We also note that the reduced $\chi^{2}$ for Mrk 9 is relatively high due to increased scatter in the data near $\sim 10$ and $30 \mathrm{keV}$, which do not correspond to any known physical features. We summarize some of the key best-fit spectral parameters for our sample in the Appendix. We did not find any sources in our sample with significant line-of-sight absorption $\left(>5 \times 10^{23} \mathrm{~cm}^{-2}\right)$, with most sources having hydrogen column densities constrained to be $<10^{22} \mathrm{~cm}^{-2}$. Figure 3 presents an example $N U S T A R$ spectrum for a potential low-cutoff candidate in our sample, 2MASX J19301380+3410495, for which we measured $E_{\text {cut }}$ to be $23_{-9}^{+29} \mathrm{keV}$.

\section{Results and Discussion}

In this section, we present limits on the high-energy cutoff, $E_{\text {cut }}$, found from spectral modeling of our sample. We then present the location of our sources on the $\theta-l$ plane for AGN coronae and discuss the implications of sources with low values of $E_{\text {cut }}$ on the heating and cooling mechanisms operating in the corona.

\subsection{Cutoff Constraints}

The distribution of lower limits on the high-energy cutoff for our sample is presented in Figure 4. The histogram shows a number of AGNs with lower limits on $E_{\text {cut }}$ below $100 \mathrm{keV}$. Typical values of $E_{\text {cut }}$ for AGNs generally range from $\sim 100$ to $300 \mathrm{keV}$ (Dadina 2007; Malizia et al. 2014; Ricci et al. 2017); we note that Gilli et al. (2007) comment that the mean value of 


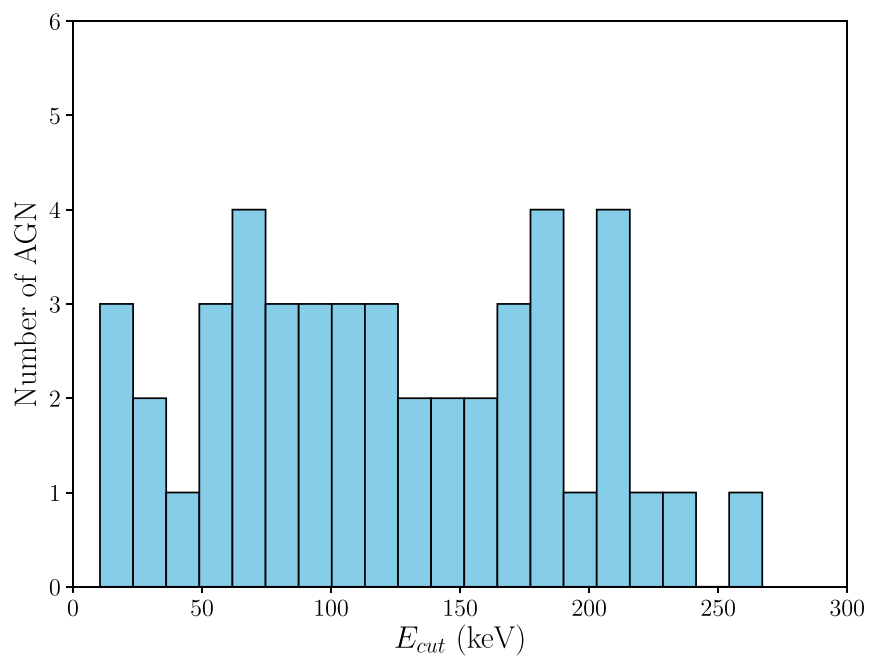

Figure 4. Distribution of lower limits on $E_{\text {cut }}$ for our Sy1 AGN sample from modeling NuSTAR data.

$E_{\text {cut }}$ for AGNs must not exceed several hundred $\mathrm{keV}$ in order to avoid overproducing the cosmic X-ray background above $100 \mathrm{keV}$.

Despite the fact that the quality of NUSTAR data in the hard $\mathrm{X}$-ray band exceeds any previous observations of our targets, the cutoff power-law model does still display a degree of degeneracy in the derived photon index $(\Gamma)$ and $E_{\text {cut }}$. In order to verify that our constraints on $E_{\text {cut }}$ are physically reasonable, in Figure 5 we compare our derived $\Gamma$ and $E_{\text {cut }}$ values to curves of constant optical depth in the $E_{\text {cut }}-\Gamma$ parameter space. The purple line in Figure 5 corresponds to theoretical constraints from Petrucci et al. (2001) for an optical depth $\tau=6$. We use the relationship derived for a slab geometry of the corona by Petrucci et al. (2001) to calculate the optical depth as a function of $\Gamma$ and $E_{\text {cut }}$ :

$$
\Gamma=\sqrt{\frac{9}{4}+\frac{511 \mathrm{keV}}{\tau k T_{e}(1+\tau / 3)}}-\frac{1}{2} .
$$

AGN coronae are typically thought to be optically thin ( $\tau<1$; Zdziarski 1985; Stern et al. 1995), though some have been constrained to $\tau \sim 3$ based on high-quality NuSTAR data (e.g., Baloković et al. 2015; Kara et al. 2017; Tortosa et al. 2017). Combinations of $\Gamma$ and $E_{\text {cut }}$ that correspond to $\tau>6$ can be considered to result from a degeneracy between model parameters, and therefore are unphysical. With this particular assumption we suspect that for three targets our results may be unrealistic; if, for example, $\tau<10$ is chosen, then no targets fall in this category. However, sources lying near or below the line with $\tau=6$ were not removed from our sample, as the limited NUSTAR data quality with a short $20 \mathrm{ks}$ exposure does not rule out physically reasonable values of the photon index.

We investigate the presence of model degeneracies in the sources with the lowest measured $E_{\text {cut }}$ constraints (2MASX $\mathrm{J} 19301380+3410495$ and 1RXS J034704.9-302409) by exploring the $E_{\mathrm{cut}}-\Gamma$ parameter space in XSPEC. Figure 6 shows the contour plots of the photon index against the highenergy cutoff for these sources. While there is some degree of degeneracy between these two parameters, the value of $E_{\text {cut }}$ is constrained to low values over the range of physically reasonable photon index values at the $68 \%$ confidence level.

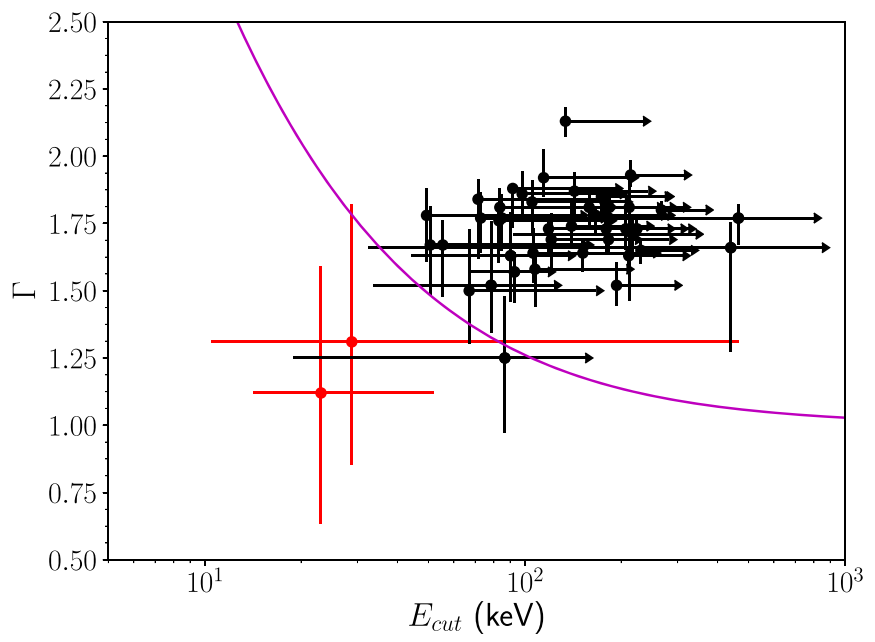

Figure 5. $E_{\text {cut }}$ vs. photon index $\Gamma$ for our sample. Points in red denote candidate sources with low coronal high-energy cutoffs for which both upper and lower limits on $E_{\text {cut }}$ were measured. The purple line corresponds to theoretical constraints from Petrucci et al. (2001) for $\tau=6$.
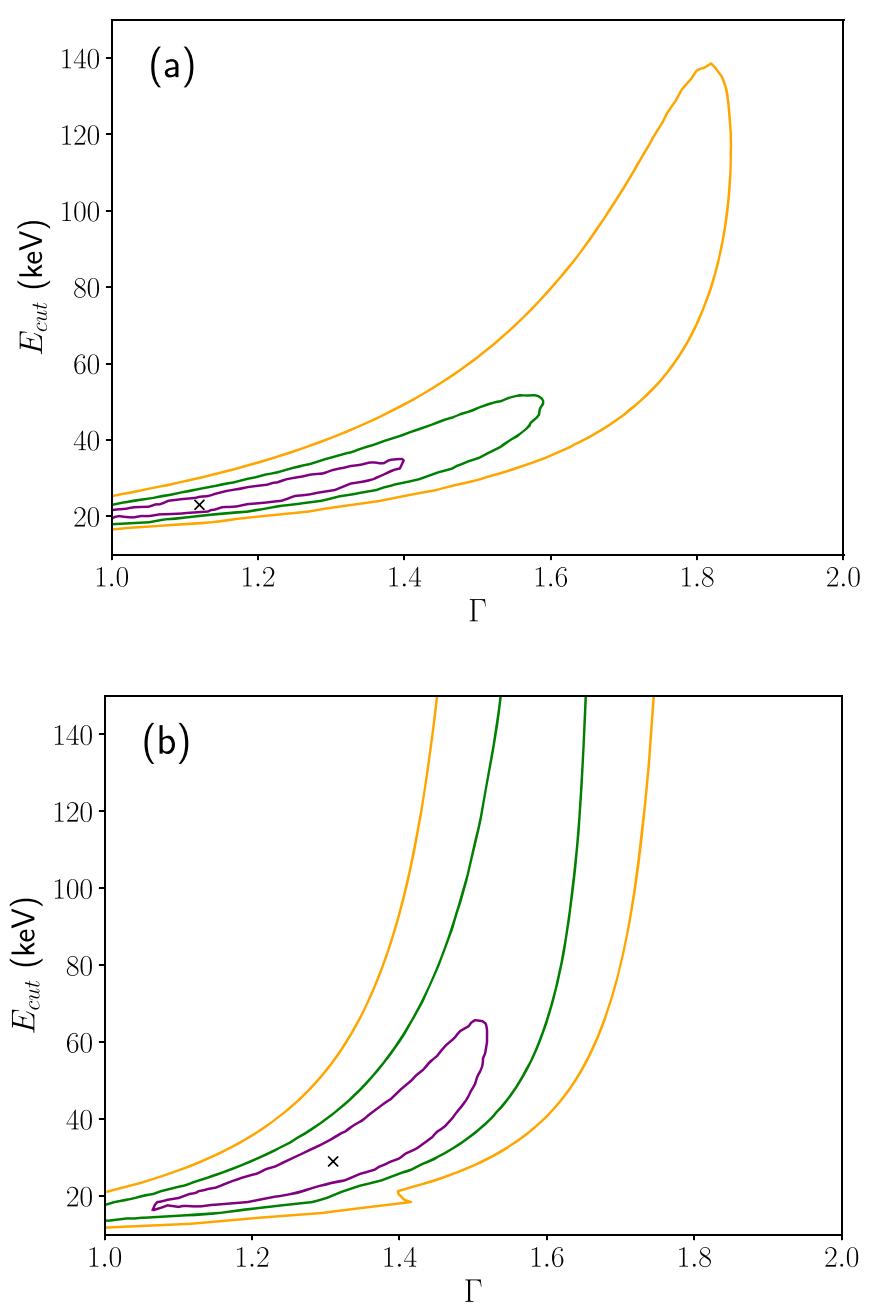

Figure 6. $E_{\text {cut }}-\Gamma$ contour plots for NuSTAR observations of the candidate lowcutoff Sy1s (a) 2MASX J19301380+3410495 and (b) 1RXS J034704.9-302409. The solid purple, green, and yellow contours correspond to the $68 \%, 90 \%$, and $99 \%$ confidence levels, respectively. The black cross represents the best-fit values of the parameters from applying the relevant model given in the Appendix. 


\subsection{The $\theta-l$ Plane}

In constructing the observational $\theta-l$ plane, we convert from $E_{\text {cut }}$ to the coronal temperature using $k_{\mathrm{B}} T_{e}=E_{\text {cut }} / 2$ (Petrucci et al. 2001). In calculating $l$, we assume a conservative value of $10 R_{g}$ for the coronal radius $R$, as adopted in Fabian et al. (2015), as the majority of the sources in our sample lack the required X-ray reflection modeling or reverberation measurements to place constraints on coronal size. We estimated the source luminosity $L$ from the flux in the $0.1-200 \mathrm{keV}$ band, which was extrapolated from the applied spectral model. We convert the unabsorbed $0.1-200 \mathrm{keV}$ flux obtained from spectral modeling to luminosity using luminosity distance values from NED. Black hole mass estimates, where available, were taken from Koss et al. (2017). The values of $M_{\mathrm{BH}}$ used in Koss et al. (2017) were obtained from a combination of broad Balmer emission line measurements, direct techniques such as X-ray reverberation mapping, and the $M_{\mathrm{BH}^{-}} \sigma_{*}$ relation of Kormendy \& Ho (2013). We have black hole mass measurements obtained from the literature for 34 of the 46 sources in our sample. For sources with no published black hole mass, we use the median black hole mass of the Sy 1-1.5 AGNs in the BAT AGN Spectroscopic Survey (BASS; Koss et al. 2017), $\log \left(M_{\mathrm{BH}} / M_{\odot}\right)=7.97 \pm 0.52$.

We note that the precise location of AGNs on the $\theta-l$ plane is dependent on general relativistic effects, such as gravitational redshift and light bending. Processes such as light bending introduce inclination-dependent corrections to $l$. These corrections depend on the geometry of the corona, which is currently highly uncertain. Therefore, due to the large uncertainties associated with model-based relativistic corrections, we do not include general relativistic effects here.

Figure 7 presents the location of our sources on the $\theta-l$ plane, in addition to theoretical pair lines for different coronal geometries. Runaway pair production occurs to the right of the pair lines, as described in the introduction. Modeling the corona as an isolated electron cloud, Svensson (1984) estimated the pair production line to have the analytical form

$$
l \sim 10 \theta^{5 / 2} e^{1 / \theta} .
$$

Stern et al. (1995) also computed the pair balance line for a slab and hemispherical corona, respectively, located above a reflecting accretion disk. The solid black and purple lines in Figure 7 correspond to these geometries.

Our results show that most of the AGN coronae in our sample are clustered near the lines for runaway pair production, similar to the results found by Fabian et al. (2015) for NuSTARobserved AGNs and black hole binaries. The pair lines thus appear to correspond to a physical boundary, constraining sources to that region. A few AGNs are located away from the pair line boundary, hinting at low coronal temperatures. Note that we have assumed that the corona is homogeneous and at a single temperature, whereas in reality there may be a range of temperatures. This may result in a mean temperature at a lower value due to Compton cooling (Fabian et al. 2015).

Recent detections of low coronal cutoffs have been made within the NuSTAR band, such as in Tortosa et al. (2017), Kara et al. (2017), and Xu et al. (2017). For example, Kara et al. (2017) measured $T_{e}=15 \pm 2 \mathrm{keV}$ for the narrow-line Sy1 Ark 564 , making it one of the lowest-temperature coronae observed by NUSTAR to date. Multiple explanations have been proposed for the origin of low-temperature coronae. In the case of an AGN accreting close to the Eddington limit, the stronger

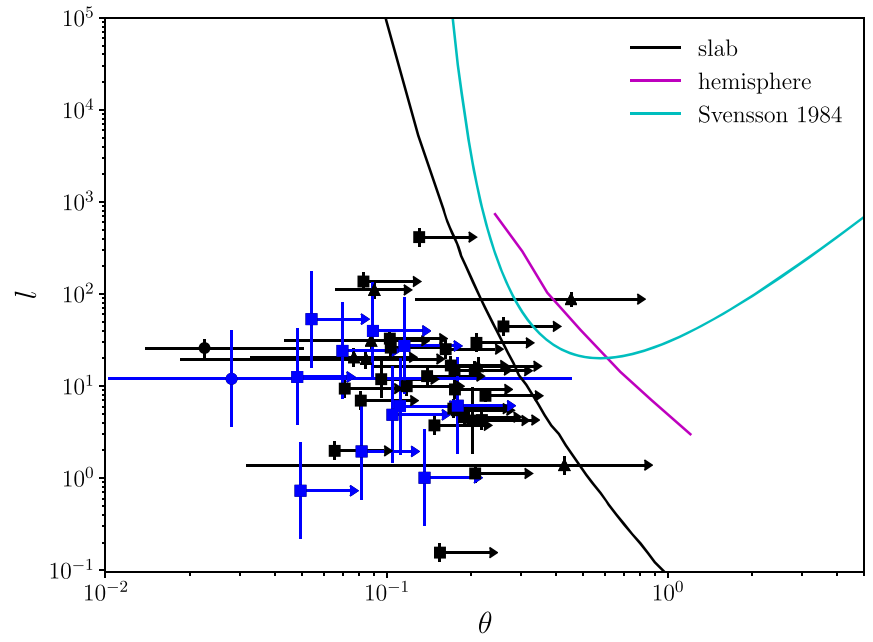

Figure 7. The $\theta-l$ plane for $N u S T A R$-observed Sy1 AGNs. Solid lines correspond to pair lines for different coronal geometries. Circled points are candidate low-cutoff sources for which both upper and lower limits on $E_{\text {cut }}$ were measured. Triangles denote sources with a best-fit value and lower limit on $E_{\text {cut }}$. Squares denote sources with only lower limits on $E_{\text {cut }}$. Blue points indicate sources for which the black hole mass was taken to be the median black hole mass of the type 1 AGNs in the BAT AGN Spectroscopic Survey (BASS; Koss et al. 2017).

radiation field may enhance Compton cooling in comparison with sub-Eddington Seyferts (Kara et al. 2017). For sources accreting well below the Eddington limit, the relatively low coronal temperatures may be attributed to highly effective cooling in some AGNs due to, e.g., high spin and the resulting higher seed photon temperature. Low temperatures may also arise from particularly weak coronal heating mechanisms, or more effective cooling due to multiple scatterings in a corona with high optical depth (e.g., Tortosa et al. 2017). Naively, when the optical depth in the corona exceeds unity, multiple inverse Compton scatterings transfer a proportionally higher fraction of the stored thermal energy to coronal luminosity. However, coronae are complex systems, and many coupled physical processes determine the electron temperature.

Another possibility is that the corona consists of a hybridized plasma, containing both thermal and nonthermal particles (e.g., Ghisellini et al. 1993; Zdziarski et al. 1993; Fabian et al. 2017). In such a system, the corona is highly magnetized and compact, and thus heating and cooling are so intense that electrons do not have time to thermalize before they are cooled by inverse Compton scattering. The presence of only a small fraction of nonthermal electrons with energies above $1 \mathrm{MeV}$ can result in runaway pair production. The cooled electron-positron pairs may redistribute their available energy, thereby reducing the mean energy per particle and decreasing the coronal temperature. Such cooling would produce a hard nonthermal tail and an annihilation feature at $511 \mathrm{keV}$. Hard X-ray data of very high quality are necessary to distinguish between a hybrid, pair-dominated plasma and cooler, fully thermal plasma incapable of pair production.

\section{Future Observations}

The $E_{\text {cut }}$ constraints presented here are based on snapshot $\sim 20 \mathrm{ks}$ NuSTAR observations of a sample of bright Sy1 galaxies and identified several sources that potentially have high-energy cutoffs within the NUSTAR band (i.e., 3-79 keV). Future work will involve performing longer exposure NUSTAR 

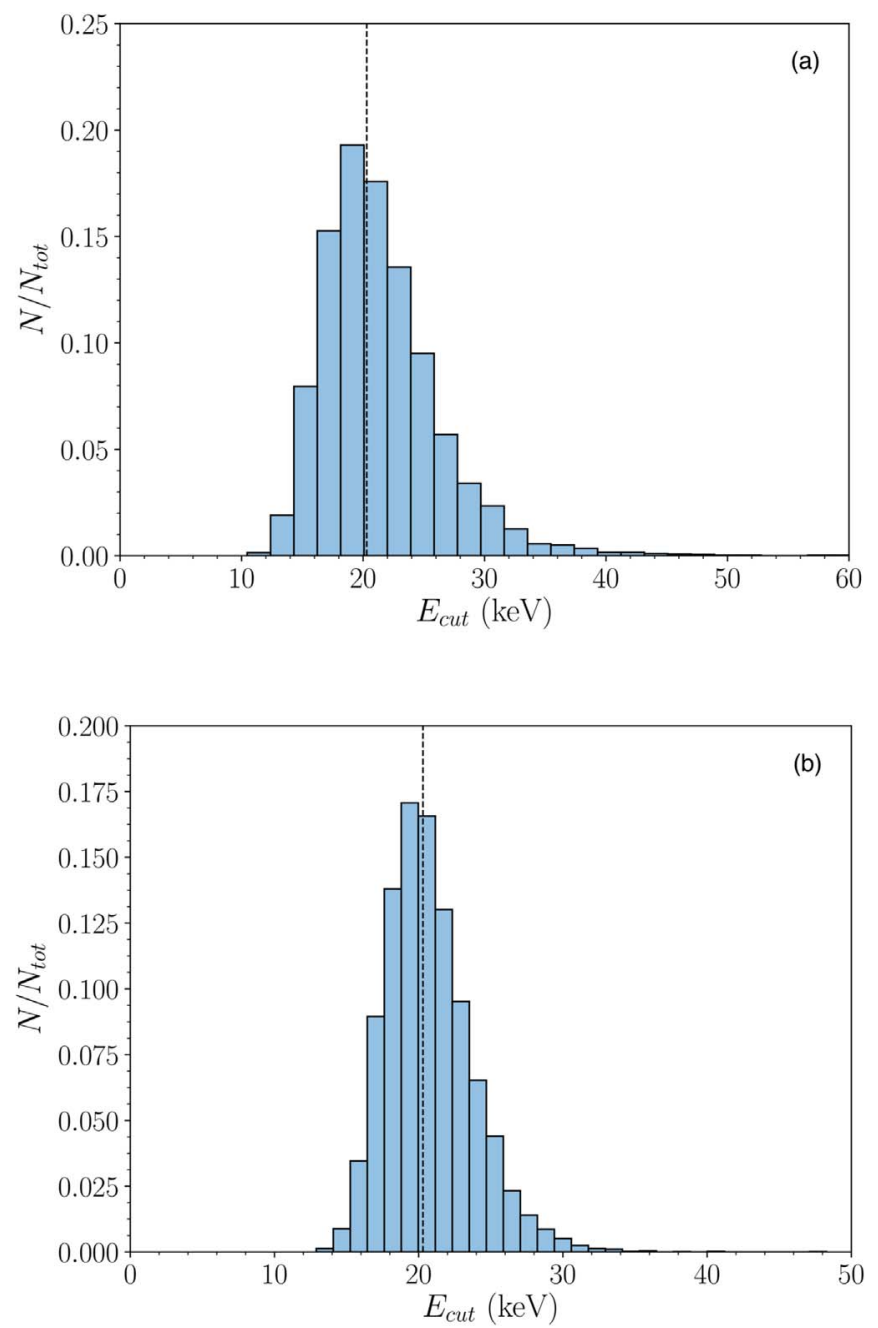

Figure 8. Distributions of $E_{\text {cut }}$ values for 5000 simulations of the NuSTAR spectrum of a candidate low-cutoff AGN, 2MASX J19301380+3410495 Dotted lines denote the input $E_{\text {cut }}$ value assumed for simulated spectra. Simulations were performed for exposure times of (a) $50 \mathrm{ks}$ and (b) $100 \mathrm{ks}$.

observations of AGNs from our sample that display hints of a low coronal cutoff, which will aid in removing model degeneracies and more tightly constrain $E_{\text {cut }}$ in order to determine the coronal temperature. In choosing AGNs from our sample for longer exposure NUSTAR observations, we performed 5000 simulations of the spectra of candidate low $E_{\text {cut }}$ AGNs from our sample in XSPEC, for exposure times of 50 and 100 ks. From the simulated spectra, we plotted distributions of the best-fit value of $E_{\text {cut }}$ found from applying an absorbed cutoff power-law model, in addition to lower limits and upper limits on $E_{\text {cut }}$. The plots in Figure 8 show distributions of values of $E_{\text {cut }}$ for one such candidate lowcutoff source, 2MASX J19301380+3410495. Table 1 summarizes the mean values of $E_{\text {cut }}$ and its lower and upper limits obtained from our simulations for some candidate low-cutoff AGNs in our sample.

The simulation results show that the distributions peak at low values of $E_{\text {cut }}$ and at similar values for both a 50 and $100 \mathrm{ks}$ exposure. We conclude from our results that a $50 \mathrm{ks}$ exposure should be sufficient to constrain the high-energy cutoff in our sample of candidate low-cutoff AGNs.
Table 1

Mean Values of the High-energy Cutoff and Its Lower and Upper Limits from Simulated NuSTAR Spectra, for Swift/BAT-selected Sy1 AGNs

\begin{tabular}{lrccc}
\hline \hline & & & $E_{\text {cut }}$ \\
Name & $\begin{array}{c}\text { Exposure } \\
\text { Time } \\
(\mathrm{ks})\end{array}$ & $\begin{array}{c}E_{\text {cut }} \\
(\mathrm{keV})\end{array}$ & $\begin{array}{c}E_{\text {cut }} \\
\text { Limit } \\
(\mathrm{keV})\end{array}$ & $\begin{array}{c}\text { Upper } \\
\text { Limit } \\
(\mathrm{keV})\end{array}$ \\
\hline 1RXS J034704.9-302409 & 50 & 92.7 & 17.1 & 118.8 \\
& 100 & 54.6 & 19.2 & 111.8 \\
2MASX J19301380 & 50 & 21.4 & 15.7 & 33.0 \\
$\quad$ +3410495 & & & & \\
& 100 & 20.7 & 16.7 & 27.0 \\
Mrk 1393 & 50 & 189.0 & 43.5 & 191.7 \\
SDSS J104326d47 & 100 & 155.0 & 45.0 & 199.7 \\
$\quad+110524.2$ & 50 & 170.4 & 46.3 & 203.2 \\
UGC 06728 & & & & \\
& 100 & 134.1 & 52.1 & 206.5 \\
& 50 & 162.6 & 59.4 & 234.8 \\
& 100 & 127.5 & 67.6 & 222.8 \\
\hline
\end{tabular}

\section{Summary}

In this work, we have investigated the coronal properties of a sample of Swift/BAT-selected Seyfert 1 AGNs that have been observed with NUSTAR. We individually modeled the NUSTAR spectra of all sources in our sample and searched for sources with hints of low coronal cutoffs, observable within the NUSTAR band. We mapped out the location of the sources in our sample on the compactness-temperature diagram for AGN coronae and found that the majority of sources lie near the boundary for runaway pair production. The pair production line corresponds to a physical boundary, constraining AGNs to that region. A few AGNs located away from the pair lines may possess low coronal temperatures; deeper $50 \mathrm{ks} N u S T A R$ observations will be performed on these sources to constrain the coronal temperature and optical depth. The detection of low coronal cutoffs may be explained via scenarios such as a strong radiation field, large optical depth, or a hybrid pair-dominated plasma. Further computations with hybrid plasma models, taking into account general relativistic effects, will help elucidate in more detail the heating and cooling mechanisms operating in the corona.

We would like to thank the referee for comments that helped improve the final version of the manuscript. We have made use of data from the NUSTAR mission, a project led by the California Institute of Technology, managed by the Jet Propulsion Laboratory, and funded by the National Aeronautics and Space Administration. We thank the NuSTAR Operations, Software and Calibration teams for support with the execution and analysis of these observations. This research has made use of the NuSTAR Data Analysis Software (NuSTARDAS) jointly developed by the ASI Science Data Center (ASDC, Italy) and the California Institute of Technology (USA). M.B. acknowledges support from NASA Headquarters under the NASA Earth and Space Science Fellowship Program (grant NNX14AQ07H) and support from the black hole Initiative at Harvard University, through the grant from the John Templeton Foundation.

Facility: NuSTAR. 


\section{Appendix}

We present the NuSTAR observation details and best-fit spectral parameters from fitting the NuSTAR data for our sample in Tables 2 and 3, respectively.

Table 2

NuSTAR Observation Details for Swift/BAT-selected Sy1 AGNs

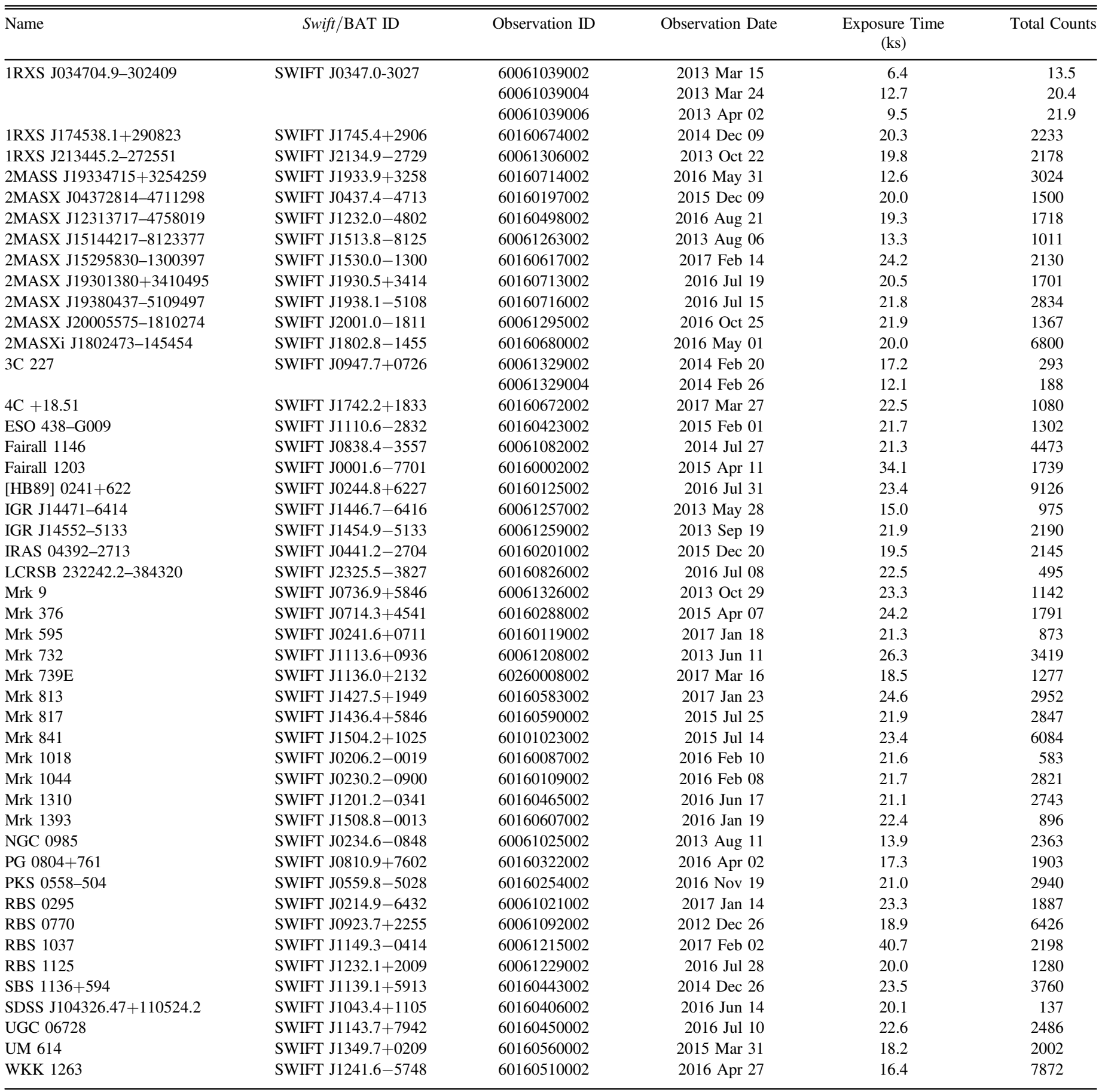


Table 3

Redshifts, Black Hole Masses, and Best-fit Spectral Parameters from Fitting NuSTAR Data for Our Swift/BAT-selected Sy1 AGN Sample

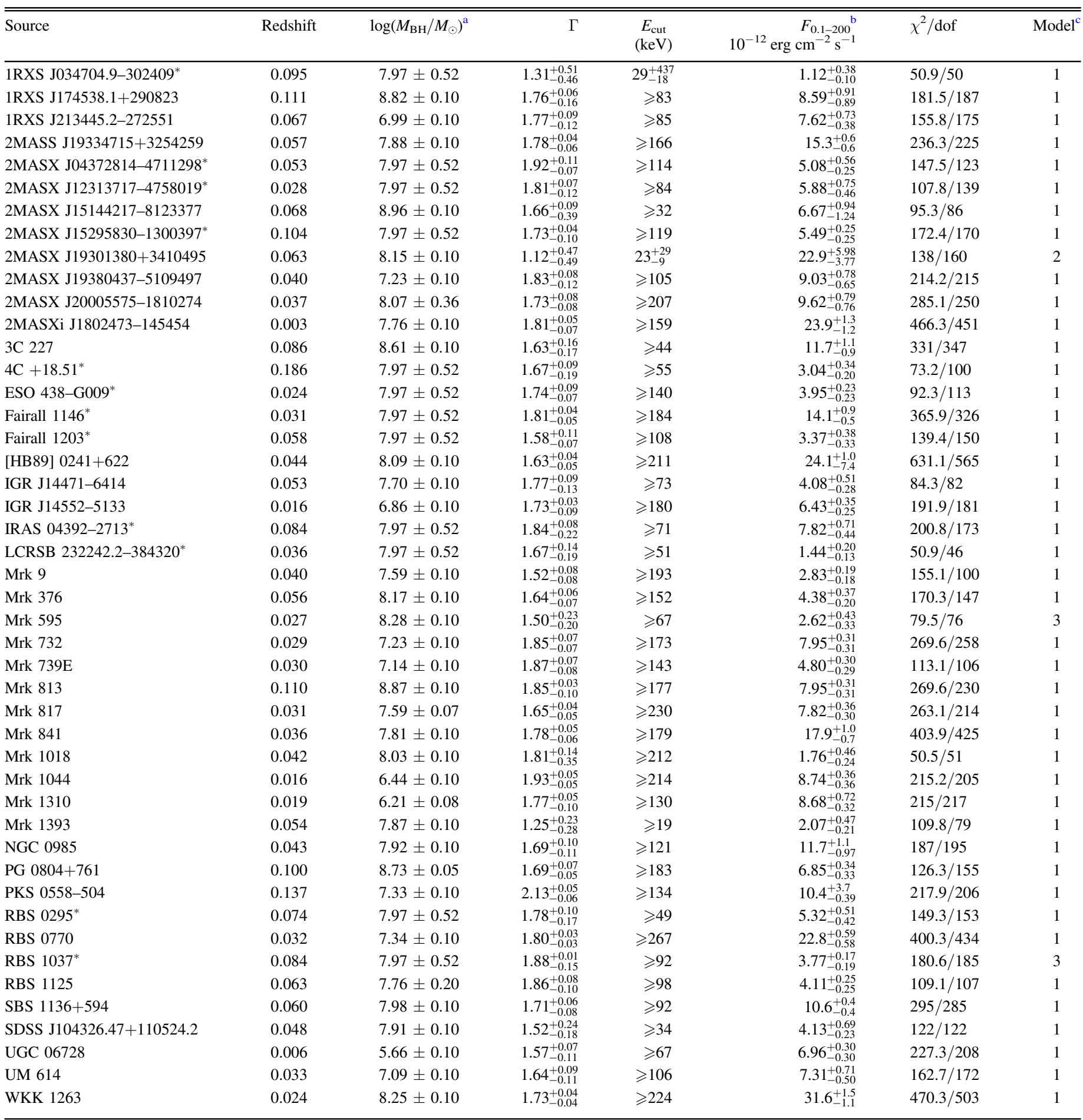

Notes. Sources marked with an asterisk ( $\left(^{*}\right.$ correspond to AGNs whose black hole masses were taken to be the median black hole mass of the type 1 AGNs in the BAT AGN Spectroscopic Survey (BASS; Koss et al. 2017).

${ }^{\mathrm{a}}$ Koss et al. (2017).

${ }^{\mathrm{b}}$ Unabsorbed $0.1-200 \mathrm{keV}$ flux extrapolated from the applied spectral model.

${ }^{c}$ Applied XSPEC models: (1) constant $\times$ TBabs $\times$ zwabs $\times$ cutoffpl, (2) constant $\times$ TBabs $\times$ zwabs $\times($ cutoffpl + pexrav), and (3) constant $\times$ TBabs $\times$ zwabs $\times$ (cutoffpl + zgauss). 


\section{ORCID iDs}

N. Kamraj 10 https://orcid.org/0000-0002-3233-2451

F. A. Harrison (1) https://orcid.org/0000-0003-2992-8024

M. Baloković (i) https://orcid.org/0000-0003-0476-6647

\section{References}

Arnaud, K. 1996, in ASP Conf. Ser. 101, Astronomical Data Analysis Software and Systems, ed. G. H. Jacoby \& J. Barnes (San Francisco, CA: ASP), 17

Ballantyne, D. R., Bollenbacher, J. M., Brenneman, L. W., et al. 2014, ApJ, 794, 62

Baloković, M., Matt, G., Harrison, F. A., et al. 2015, ApJ, 800, 62

Baumgartner, W. H., Tueller, J., Markwardt, C. B., et al. 2013, ApJS, 207, 19

Brenneman, L. W., Madejski, G., Fuerst, F., et al. 2014, ApJ, 781, 83

Dadina, M. 2007, A\&A, 461, 1209

Emmanoulopoulos, D., Papadakis, I. E., Dovčiak, M., \& McHardy, I. M. 2014 MNRAS, 439, 3931

Fabian, A. C., Lohfink, A., Belmont, R., Malzac, J., \& Coppi, P. 2017, MNRAS, 467, 2566

Fabian, A. C., Lohfink, A., Kara, E., et al. 2015, MNRAS, 451, 4375

Fabian, A. C., Zoghbi, A., Ross, R. R., et al. 2009, Natur, 459, 540

Gehrels, N., Chincarini, G., Giommi, P., et al. 2004, ApJ, 611, 1005

Ghisellini, G., Haardt, F., \& Fabian, A. C. 1993, MNRAS, 263, 9

Gilli, R., Comastri, A., \& Hasinger, G. 2007, A\&A, 463, 79

Guilbert, P. W., Fabian, A. C., \& Rees, M. J. 1983, MNRAS, 205, 593

Haardt, F., \& Maraschi, L. 1993, ApJ, 413, 507

Harrison, F. A., Craig, W. W., Christensen, F. E., et al. 2013, ApJ, 770, 103
Kalberla, P. M. W., Burton, W. B., Hartmann, D., et al. 2005, A\&A, 440, 775

Kara, E., Fabian, A. C., Cackett, E. M., Miniutti, G., \& Uttley, P. 2013, MNRAS, 430, 1408

Kara, E., García, J. A., Lohfink, A., et al. 2017, MNRAS, 468, 3489

Kormendy, J., \& Ho, L. C. 2013, ARA\&A, 51, 511

Koss, M., Trakhtenbrot, B., Ricci, C., et al. 2017, ApJ, 850, 74

Madsen, K. K., Harrison, F. A., Markwardt, C. B., et al. 2015, ApJ, 220, 8

Magdziarz, P., \& Zdziarski, A. A. 1995, MNRAS, 273, 837

Malizia, A., Molina, M., Bassani, L., et al. 2014, ApJL, 782, L25

Marinucci, A., Matt, G., Miniutti, G., et al. 2014, ApJ, 787, 83

Massaro, E., Giommi, P., Leto, C., et al. 2009, A\&A, 495, 691

Perri, M., Puccetti, S., Spagnuolo, N., et al. 2017, The NuSTAR Data Analysis Software Guide v1.9.3, https://heasarc.gsfc.nasa.gov/docs/nustar/analysis/ nustar_swguide.pdf

Petrucci, P. O., Haardt, F., Maraschi, L., et al. 2001, ApJ, 556, 716

Ricci, C., Trakhtenbrot, B., Koss, M. J., et al. 2017, ApJS, 233, 17

Rybicki, G. B., \& Lightman, A. P. 1979, Radiative Processes in Astrophysics (New York: Wiley-Interscience)

Stern, B. E., Poutanen, J., Svensson, R., Sikora, M., \& Begelman, M. C. 1995, ApJL, 449, L13

Svensson, R. 1982, ApJ, 258, 335

Svensson, R. 1984, MNRAS, 209, 175

Tortosa, A., Marinucci, A., Matt, G., et al. 2017, MNRAS, 466, 4193

Verner, D. A., Ferland, G. J., Korista, K. T., \& Yakovlev, D. G. 1996, ApJ, 465,487

Wilms, J., Allen, A., \& McCray, R. 2000, ApJ, 542, 914

Xu, Y., Baloković, M., Walton, D. J., et al. 2017, ApJ, 837, 21

Zdziarski, A. A. 1985, ApJ, 289, 514

Zdziarski, A. A., Poutanen, J., \& Johnson, W. N. 2000, ApJ, 542, 703

Zdziarski, A. A., Zycki, P. T., \& Krolik, J. H. 1993, ApJL, 414, L81 